HOCKING REPLIES - I did, in fact, consider disposal costs - see, for example, ref. 4 in ref. 1 (cited here as ref. 2). Van Eijk et al. have considered the bulkier packaging used for shipping and cleaning agents for ceramic cups, plus the larger landfill volume required by a reusable cup on eventual discard ${ }^{2}$. From this information, they obtained break-even values for the landfill volume consumed by porcelain/ polystyrene and porcelain/paper cup pairs of 125 and 99 , respectively. In other words, one could use once and discard 125 polystyrene cups before the landfill volume consumed would equal the landfill volume consumed by 125 uses of a porcelain cup. Fewer uses than these gave lower landfill volumes per use for the disposable cups than for the porcelain cup. At more than 125 uses of the porcelain cup the landfill volume occupied per use gradually dropped relative to the disposable options so that at 3,000 uses this required a total of 2.4 litres whereas the polystyrene and paper options required 9.1 and 11 litres, respectively.

Reuse of cups, whether reusable or not, expends more resources than recycling. This factor, plus aesthetics, keeps reusable cups dominant in beverage service. Polystyrene (or other plastics) and paper can be materially useful in either recycling or energy recovery programmes, the next levels of resource use. Glass can be recycled, saving at least landfill space, as the energy required to transport glass locally and remelt it is scarcely less than that of transporting and melting the raw materials. But ceramic cups that have reached the end of their useful life have no recycle or energy-recovery attributes.

Some readers have written to me direct to question the energy costs of initial cup delivery, among other factors. I did include delivery energy in my calculations ${ }^{1}$. Non-energetic costs such as destroyed natural habitat are difficult to quantify for diverse technologies ${ }^{3.4}$. But the combined habitat impacts of a large-scale china clay processing facility and chinaware firing line are at least of a similar order to those of a pulp mill or an oil-refinery complex. The air pollution impact of the porcelain option is much higher than paper or polystyrene at a few uses ${ }^{2}$. At 3,000 uses, this impact per use drops to about the same for porcelain and polystyrene and a slightly higher impact value for paper. Paper and polystyrene produce only about one-third of the water pollution impact of porcelain per use because of the need to wash reusable cups.

These details from our own and other studies confirm that there are situations,

\footnotetext{
1. Hocking, M. B. Nature 369, 107 (1994).

2. van Eijk, J. et al. Reusable Versus Disposable. (Ministry of Housing. Physical Planning and Environment, Zoetermeer. 1992).

3. Hocking, M. B. Env. Man. 15, 731-747 (1991)

3. Hocking, M. B. Env. Man, 15, 731-747 (1991)
4. Hocking, M. B. Science 251, 504-505 (1991)

5. Hocking, M. B. Env. Man. (in the press).
}

when only a low number of cup-use cycles can be anticipated, where use of disposable cups could be more efficient and less harmful to the environment than use of any of the reusable cups. (The complete version of the calculations used in ref. 1 is available in ref. 5 , which should be published in December.)

Martin B. Hocking

Department of Chemistry,

University of Victoria,

Victoria, British Columbia,

Canada V8W $3 P 6$

\section{Avalanche survival chances}

SIR - Falk et al. ${ }^{1}$ in their interesting Scientific Correspondence demonstrated the high risk of death due to burial by avalanches, and the importance of avoiding them. I cannot fully agree, however, with their conclusion that the depth of burial has no direct influence on survival chances.

I have analysed mountain sports accidents in Austria since 1986. In cases of

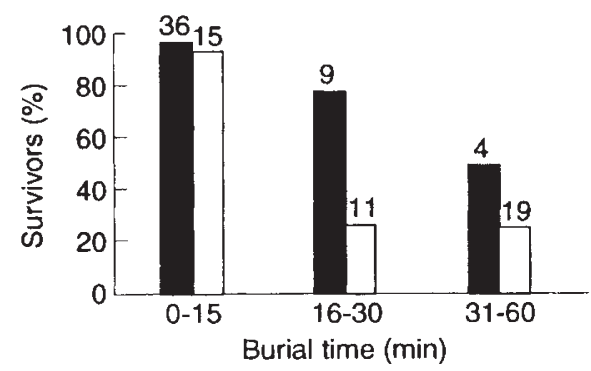

FIG. 1 Black bars, percentage of survivors buried at, or at depths less than, the overall median burial depth $(50 \mathrm{~cm})$; white bars, those buried deeper than this. Figures above the bars indicate the number of buried skiers within each subgroup.

burial by avalanches, I recorded the rescue outcome, burial time and depth of burial. In a period of 7 years $(1986-92)$, 774 people were caught in avalanches; of these, 380 were buried completely (the entire body covered by snow) and 148 were killed. Of the totally buried skiers, only 94 were known reliably to have been buried not more than 1 hour; of these, 28 (30 per cent) were dead on extrication.

The percentage of survivors in three pre-defined time periods are presented for two subgroups which represent those buried either deeper than, or at or less than, the overall median burial depth $(50$ $\mathrm{cm}$ ) (Fig. 1). Burial depths were $62 \pm 43$ $\mathrm{cm}$ (mean \pm s.d.) up to a burial time of 15 $\min , 104 \pm 61 \mathrm{~cm}$ at a burial time of $16-30$ $\mathrm{min}$, and $115 \pm 42 \mathrm{~cm}$ at a burial time of $31-60 \mathrm{~min}$. For those buried for $15 \mathrm{~min}$ or more, people buried less deeply had a greater chance of survival than those

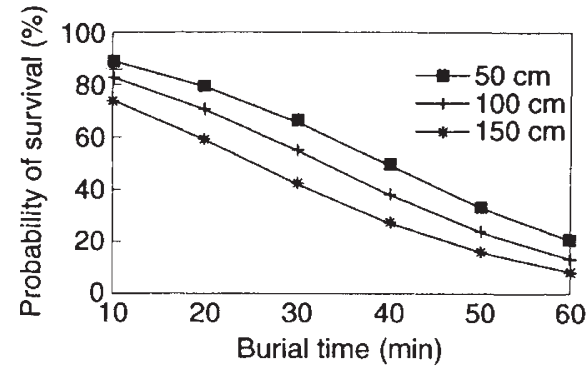

FIG. 2 Time- and depth-dependent probability of survival based on the result of logistic regression.

buried more deeply.

The question arises whether the reduced survival chance at greater depth is the result of a longer extrication time, or whether an additional direct effect of burial depth exists. Analysing the data, I was able to demonstrate a time-dependent and time-independent effect of burial depth on the chance of survival. A correlation exists between burial time and burial depth $(r=0.5, P<0.001)$, primarily due to the longer extrication time (timedependent effect). I tested the direct influence of depth of burial on survival by applying stepwise logistic regression to rescue outcome (dependent variable) in relationship to burial time and depth (independent variables). Burial time entered the model at step 1 and depth of burial at step 2, and its presence effected a significant improvement compared with step 1 (improvement $\chi^{2}=3.76, P=0.05$ ). The resulting model for the probability of survival is:

$$
\frac{\mathrm{e}^{3.232-0.06796 t_{\mathrm{h}} \cdots 0.01034 d_{\mathrm{h}}}}{1+\mathrm{e}^{3.232 \cdots 0.06796 t_{\mathrm{h}}-0.01034 d_{\mathrm{h}}}}
$$

where $t_{\mathrm{b}}$ is burial time and $d_{\mathrm{b}}$ is burial depth. The probability of survival at various burial depths based on the result of logistic regression is presented in Fig. 2. The time-independent effect of burial depth on the chance of survival is probably brought about by increased traumatization and thorax compression at a greater depth. This assumption is also supported by Stalsberg et al. ${ }^{2}$, who showed that the immediate cause of death of avalanche victims in most cases was general body compression with acute respiratory and circulatory failure. Hence, when an avalanche begins, all measures helping to keep the skier on the surface increase the chance of survival.

\section{Martin Burtscher}

Health Section of the Austrian

Alpine Club and

Austrian Society for Mountain Medicine, Bauerngasse 7, A-6065 Thaur,

Austria

\footnotetext{
1. Falk, M., Brugger, H. \& Adler-Kastner, L. Nature $\mathbf{3 6 8}, 21$ (1994)

2. Stalsberg, H. etal. Virchows Arch path. Anat. Histopath 414, 415-422 (1989)
} 